\title{
A reproducible new model of focal ischemic injury in the marmoset monkey: MRI and behavioural follow-up
}

Alice Le Friec*, Franck Desmoulin*, Boris Demain, Carole Davoust, Lorenne Robert, Tanguy Duval, Florence Rémy ${ }^{1}$, Carla Cirillo and Isabelle Loubinoux ${ }^{\#}$

ToNIC, Toulouse Neurolmaging Center, Inserm, Université de Toulouse, UPS, France

${ }^{1}$ CerCo, Centre de Recherche Cerveau \& Cognition, CNRS, Université de Toulouse, UPS, France

*These authors contributed equally

"Correspondence to:

Dr Isabelle Loubinoux, UMR 1214 -Inserm/UPS- ToNIC Toulouse Neurolmaging Center CHU Purpan - Pavillon Baudot Place du Docteur Baylac 31024 Toulouse - Cedex 3 Tel: 33(0)5 62746183 Fax: 33(0)5 62746163

Email: isabelle.loubinoux@inserm.fr http:/www.tonic.inserm.fr/ 


\section{ABSTRACT}

Background \& Aims: Ischemic stroke mostly affects the primary motor cortex and descending motor fibres, with consequent motor impairment. Pre-clinical models of stroke with reproducible and long-lasting sensorimotor deficits in higher-order animals are lacking. We describe a new method to induce focal brain damage targeting the motor cortex to study damage to the descending motor tracts in the non-human primate.

Methods: Stereotaxic injection of malonate into the primary motor cortex produced a focal lesion in middle-aged marmosets (Callithrix jacchus). Assessment of sensorimotor function using a neurological scale, and testing of forelimb dexterity and strength lasted a minimum of 12 weeks. Lesion evolution was followed by Magnetic Resonance Imaging (MRI) at 24h, 1 week, 4 and 12 weeks post-injury and before sacrifice for immunohistochemistry.

Results: Our model produced consistent lesions of the motor cortex, subcortical white matter and caudate nucleus. All animals displayed partial spontaneous recovery with long lasting motor deficits of force ( $54 \%$ loss) and dexterity ( $\approx 70 \%$ loss). Clearly visible T2 hypointensity in the white matter was observed with MRI and corresponded to areas of chronic gliosis in the internal capsule and lenticular fasciculus.

Conclusions: We describe a straightforward procedure to reproducibly injure the motor cortex in the marmoset monkey, causing long lasting motor deficits. The MRI signature reflects Wallerian degeneration and remote injury of corticospinal and corticopontine tracts, as well as subcortical motor loops. Our model may be suitable for the testing of therapies for poststroke recovery, particularly in the chronic phase.

KEYWORDS: stroke, marmoset, Magnetic Resonance Imaging, malonate, sensorimotor deficit 


\section{INTRODUCTION}

Ischemic stroke frequently disrupts cerebral circulation within the Middle Cerebral Artery, causing damage to supplied territories such as the primary motor cortex and descending motor fibres [1], key areas controlling motor function. In patients, motor cortex involvement and corticospinal tract (CST) damage have been shown to negatively impact motor recovery [2], [3]. Recovery is near maximal after 6 months to one year, highlighting the need for novel therapies targeting the chronic phase [4], [5]. Progress in this direction is limited by a lack of valid preclinical models that accurately reproduce the effects of ischemic stroke. The revised STAIR (Stroke Therapy Academic Industry Roundtable) guidelines recommend the use of higher-order animals such as non-human primates in preclinical studies of stroke [6]. The common marmoset (Callithrix jacchus) offers a valid compromise in terms of proximity to humans and ease of handling. Marmosets have a brain size 4 times larger than that of rats, with a higher white to grey matter ratio. Marmosets also display a rich behavioural repertoire and possess a high degree of forelimb dexterity, crucial for the study of motor deficits after stroke.

Several marmoset models of stroke have been described. They most often involve permanent or transient middle cerebral artery occlusion (MCAo). Occlusion by electrocoagulation of the MCA requires a large craniotomy in marmosets, and produces extensive brain damage [10], [11]. MCAo can also be induced using Endothelin-1, a potent vasoconstrictor agent. However, in this case, the duration of ischemia cannot be controlled [12]. Another approach is the intraluminal thread approach to MCAo, which allows permanent or transient occlusion through tight control of ischemia duration [13], [14].. The lesions produced by permanent occlusion are often extensive and injure premotor and parietal regions as well as sub-cortical nuclei, causing severe deficits [10], [14]. Importantly, the primary motor cortex is not consistently damaged by MCAo. Finally, due to individual differences in vascular anatomy and reperfusion events, the anatomical and functional consequences of MCAo may vary significantly across subjects. Although consistent with the clinical situation, high variability can be a disadvantage for the evaluation of new therapies for stroke. Indeed, in this case, comparable pre-treatment groups are required so that fewer animals are needed to measure statistically significant effects. Infarction produced by photothrombosis is another vascular model that led to small cortical lesions of reproducible 
location and lasting motor deficits (evaluated up to one month) in marmosets [15]. However, lesions produced by photothrombosis did not reach the basal ganglia and deficits were not assessed in the long-term in this study. Vascular models have been of great importance to study the acute effects of ischemic stroke [7] as well as the effect of neuroprotective therapeutic strategies [8], [9]. Non-vascular models may be of particular interest for the testing of therapeutic strategies focusing on brain repair in the context of established chronic lesions.

To our knowledge, non-vascular models of stroke for the study of brain repair therapeutic strategies have not yet been described in the marmoset monkey. Our goal was to induce reproducible focal brain damage targeting the motor cortex and to study long-term damage to the descending motor tracts in order to provide a valid model of ischemic stroke in the non-human primate. We used stereotaxic injection of malonate into the primary motor cortex of the adult marmoset. This mitochondrial toxin is a competitive inhibitor of the succinate dehydrogenase enzyme, a key protein of the mitochondrial electron transport chain. Cell death occurs through necrosis and apoptosis due to the synergistic effects of energy failure and subsequent excitotoxicity leading to brain damage similar to that observed after an ischemic insult in humans [16]-[22].

This approach has been previously validated in rats by our team, and has proven to be a survivable procedure that leads to long-term functional deficits whether the lesion involves the cortex and striatum or the internal capsule [17], [22], thus suitable for testing of stem cellbased therapies [18], [21]. However, a major limitation of the rodent model in the context of post-stroke therapy testing is the total absence of measurable dexterity recovery [17], [22]. In addition, the anatomy of brain structures involved in motor control, particularly the basal ganglia and subcortical motor loops, are dissimilar to the human brain. The objective of the present translational study was to adapt the malonate model of motor cortex lesion to a nonhuman primate species that displays more dexterous abilities and a brain anatomy closer to humans. We aimed to characterize the long-term anatomical and functional consequences of the motor cortex lesion in marmoset monkeys.

\section{MATERIALS AND METHODS}

\section{Animals}


Thirteen male ( 290 to $450 \mathrm{~g}$, aged 47 to 95 (57 \pm 8) months) and seven female (290 to $420 \mathrm{~g}$, aged 47 to 67 (63 \pm 15 ) months) laboratory-bred marmoset monkeys (Callithrix jacchus) were used. Animals were excluded if they had missing digits, or if they were otherwise unable to use a test apparatus for whatever reason. All animals were maintained and treated according to European regulations (EU Directive 2010/63). Our protocol was approved by the Direction départementale de la Protection des Populations de la Haute-Garonne and the Comité d'éthique pour l'expérimentation animale Midi-Pyrénées (authorization $n^{\circ} 31125507$ and $n^{\circ}$ B3155501). All efforts were made to minimize the number of animals used and the suffering they experienced.

\section{Stereotaxic injection of malonate}

Animals were food restricted for $12 \mathrm{~h}$ before surgery. Ten minutes before anaesthesia, animals received intramuscular (IM) injection of glycopyrrolate $\left(20 \mu \mathrm{g} / \mathrm{kg}\right.$, Robinul- ${ }^{\circledR}$, Vetoquinol, Lure, France) to avoid excessive salivation. Anaesthesia was induced with alfaxolone $(7 \mathrm{mg} / \mathrm{kg}$, Alfaxan $^{\circledR}$, Worcestershire, UK). Marmosets were premedicated with IM injections of a longacting antibiotic, (oxytetracycline, $20 \mu \mathrm{g} / \mathrm{kg}$, Terramycine longue action ${ }^{\circledR}$, Pfizer, Paris, France), analgesic (buprenorphine, $25 \mu \mathrm{g} / \mathrm{kg}$, Vétergesic ${ }^{\circledR}$, Reckitt Benckiser Healthcare, Danson, UK) and an anti-inflammatory drug (methylprednisolone, $5 \mathrm{mg} / \mathrm{kg}$, Solumedrol ${ }^{\circledR}$, Zoetis), to avoid excess oedema. Anaesthesia was maintained with isoflurane (1-3\%) in $\mathrm{O}_{2}(100 \%)$. Oxygen saturation was maintained above $95 \%$, rectal temperature was maintained close to $37.5^{\circ} \mathrm{C}$ with a heating pad. If respiratory distress occurred, 2 drops of doxapram (Dopram ${ }^{\circledR}$, Vetoquinol) were administered intra-nasally ( $n=4)$.

Animals were placed in a stereotaxic frame, heads fixed with ear-bars. Under aseptic conditions, the scalp was incised and a small burr hole $(1 \times 1 \mathrm{~mm})$ was drilled under cooling saline. Dura was incised and $8 \mu \mathrm{L}$ of malonate (Sigma) at a concentration of 3M in Dulbecco's Phosphate Buffer Solution (DPBS, Gibco) ( $n=18$ ) or DPBS ( $n=2$, sham group) was injected using a $10 \mu \mathrm{L}$ syringe (Hamilton 701N Series, Phymep, Paris, France, $0.485 \mathrm{~mm}$ gauge needle) connected to a micropump (injection rate: $1 \mu \mathrm{L} / \mathrm{min}$ ). The stereotaxic coordinates targeted the primary motor cortex (AP $+6 \mathrm{~mm}, \mathrm{ML}+/-4.5 \mathrm{~mm}, \mathrm{SI}+5 \mathrm{~mm}$ from Bregma, Stereotaxic Atlas of the Marmoset Brain [23]) of the hemisphere controlling the dominant forelimb. After the 
injection, the needle was left in place for an additional 5 minutes to avoid backflow. Skin was resewn with 3.0 thread (Vicryl), and animals were allowed to recover from anaesthesia before returning to their home cages. Animals were given access to water and soft food, hand fed if necessary, and monitored daily throughout convalescence. Usually, marmosets could move and feed without assistance after 1-3 days.

\section{Post-operative care}

The drilling of the skull may cause moderate pain, managed if necessary with Tolfedine ${ }^{\circledR}$ (Vetoquinol) (2 mg/kg/day, IM). In the case of more severe discomfort linked to post-operative oedema, animals were given methylprednisolone twice daily $(5 \mathrm{mg} / \mathrm{kg}, \mathrm{IM}$ ) for 24-36 hours. One animal presented epileptic seizures five days after surgery, which were managed with Levitiracetam (Keppra, $8.3 \mathrm{mg} / \mathrm{kg}$, IM).

\section{Behavioural assessment}

Before surgery, animals were trained 3-5 times a week for 2 months on testing apparatus until maximum performance was consistently observed. The apparatus was attached to the homecage and no dietary restriction was necessary. Rewards were small pieces of gingerbread. After brain lesion, animals were tested 3 times a week for the first month, then three times during the first week of the following months until the end of the protocol (minimum 3 months). The limb with consistently higher scores in the staircase and dynamometric pull test was considered the dominant forelimb. The dominant forelimb was the future contralesional (impaired) forelimb, whereas the non-dominant forelimb was the future ipsilesional (unimpaired) forelimb.

\section{Hill and valley staircase}

To evaluate forelimb dexterity, we used the staircase test by Marshall and Ridley [10] which we slightly modified. This consists of a clear Plexiglas apparatus that attaches to the homecage and has two openings (one for the right arm and one for the left arm), each giving access to a 'staircase' with five steps. The animal passes its arm through the opening and retrieves small treats placed onto the steps, with the first step being the easiest to reach and the fifth step the hardest. We created grooves in each step of the staircase to increase test difficulty. Marmosets retrieved a reward on each step (1 to 5 points given per step, 15 points maximum), and unlike in Marshall and Ridley, we awarded points if the reward was dropped to a lower 
step (i.e. the points from the lower step). As the Hill staircase does not separate motor from visuospatial deficits, we also used the Valley staircase. In the Valley version, the contralesional visual hemi-field (potentially neglected) is reached by the ipsilesional (unaffected) forelimb and conversely, through a single opening, allowing the detection of spatial deficits. Animals were allowed 300 seconds/forelimb to retrieve all the rewards.

\section{Dynamometric pull test}

While the study was ongoing, we developed a novel quantitative system for the measurement of forelimb strength. The apparatus was made of Plexiglas and was fixed to the home-cage during sessions. A detailed diagram of the Dynamometric Pull Test (DPT) is available upon request. Animals were trained for two months to reach through an opening and grip a small handle attached to a spring and dynamometer. When the marmosets pulled on the handle, small treats were released and the force applied to the handle was measured (Newtons). Five levels of increasing difficulty (from 1 to $6 \mathrm{~N}$ ) were provided by the DPT, and animals were given 300 seconds/forelimb to complete the test.

\section{Neurological scale}

We adapted a marmoset neurological scale used to evaluate deficits after spinal cord injury (table 1), which was slightly modified to include use of test apparatus (staircases, DPT), chewing of food and evaluation of paw sensitivity [24]. Left and right sides of the body were evaluated separately, with 22 points maximum attributed per side. Marmosets were observed during the testing sessions and scored twice a week by two experimenters.

\section{MOTRICITY (maximum 10 points per side)}

A. Upper limbs (maximum 7 points per side)

\begin{tabular}{|l|l|c|}
\hline Use of dynamometric pull test & Does not reach hand through opening & +0 \\
\hline & Reaches hand through opening & +1 \\
\hline & Grips handle & +2 \\
\hline Use of staircase test & Pulls on handle & +0 \\
\hline & Does not reach hand through opening & +1 \\
\hline & Reaches with paretic hand if cheating is prevented & +2 \\
\hline
\end{tabular}




\begin{tabular}{|c|c|c|}
\hline \multirow[t]{2}{*}{ Capacity to reach } & Abnormal & +0 \\
\hline & Normal & +1 \\
\hline \multirow[t]{2}{*}{ Movement on cage floor } & Abnormal & +0 \\
\hline & Normal & +1 \\
\hline \multicolumn{3}{|c|}{ B. Hindlimb (maximum 3 points per side) } \\
\hline \multirow[t]{3}{*}{ Standing position } & Not possible & +0 \\
\hline & Possible but unstable & +1 \\
\hline & Normal & +2 \\
\hline \multirow[t]{2}{*}{ Movement on cage floor } & Abnormal & +0 \\
\hline & Normal & +1 \\
\hline \multicolumn{3}{|c|}{ SENSITIVITY (maximum 7 points per side) } \\
\hline \multicolumn{3}{|c|}{ A. Upper limbs (maximum 5 points per side) } \\
\hline \multirow[t]{3}{*}{ Weight bearing on cage floor } & Hand does not touch cage floor & +0 \\
\hline & Abnormal contact between hand and cage floor & +1 \\
\hline & Normal & +2 \\
\hline \multirow{3}{*}{$\begin{array}{l}\text { Frequency of contact between hand and cage } \\
\text { floor }\end{array}$} & No contact & +0 \\
\hline & Contact is less frequent than with unaffected hand & +1 \\
\hline & $\begin{array}{l}\text { Equal frequency of contact with cage floor } \\
\text { between the two hands }\end{array}$ & +2 \\
\hline \multirow[t]{2}{*}{ Sensitivity to a light prick sensation* } & Abnormal & +0 \\
\hline & Normal & +1 \\
\hline \multicolumn{3}{|c|}{ B. Hindlimb (maximum 2 points per side) } \\
\hline \multirow[t]{2}{*}{ Weight bearing on cage floor } & Abnormal contact with cage floor & +0 \\
\hline & Normal & +1 \\
\hline \multirow[t]{2}{*}{ Sensitivity to a light prick sensation } & Abnormal & +0 \\
\hline & Normal & +1 \\
\hline \multicolumn{3}{|c|}{ FACE (maximum 2 points per side) } \\
\hline \multirow[t]{3}{*}{ Chewing of food } & Does not eat & +0 \\
\hline & Abnormal & +1 \\
\hline & Normal & +2 \\
\hline \multicolumn{3}{|c|}{ TRUNK (maximum 1 point per side) } \\
\hline \multirow[t]{2}{*}{ Position of trunk when sitting** } & Asymmetrical posture & +0 \\
\hline & Normal & +1 \\
\hline \multicolumn{3}{|c|}{ JUMPS (maximum 3 points per side) } \\
\hline Jumps between sides of cage $\mathrm{e}^{* * *}$ & No jumps & +0 \\
\hline
\end{tabular}




\begin{tabular}{|l|l|c|}
\hline & Fewer or shorter jumps & +1 \\
\hline & Normal & +2 \\
\hline
\end{tabular}

Table 1. Adapted marmoset neurological scale

* Sensitivity was tested by distracting the monkey and pricking the palm of the hand with a toothpick.

**Position of the trunk was evaluated by careful observation of the monkey when it was sitting in front of the test apparatus.

***Points were lost on the score for both sides of the body.

\section{MRI}

Animals underwent longitudinal MRI evaluation before and $24 h$, one week $(n=5)$, four weeks and twelve weeks ( $n=12$ animals at the twelve-week time point) after surgery, under general anaesthesia. The scanner was a 3 Tesla Achieva (Philips) MRI scanner fitted successively with one of the following coils: a human knee coil (a) or human wrist coil (b) in which the animals lay supine under chemical anaesthesia (alfaxolone, $18 \mathrm{mg} / \mathrm{kg}$ ) or a solenoid coil (c) in which the animals were placed in the prone position under chemical and gaseous anaesthesia (alfaxolone, $7 \mathrm{mg} / \mathrm{kg}$ and isoflurane $1.5-3 \%$ ).

A high-resolution 3D T2-weighted sequence used the following parameters (a) using the

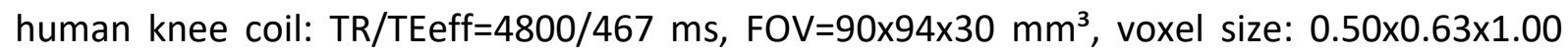
$\mathrm{mm}^{3}$, number of slices acquired $=30$, scan duration=21 minutes, number of animals=2. (b) using the human wrist coil: TR/TEeff $=4800 / 467 \mathrm{~ms}$, FOV $=90 \times 94 \times 30 \mathrm{~mm}^{3}$, voxel size: $0.50 \times 0.63 \times 1.00 \mathrm{~mm}^{3}$, number of slices acquired $=30$, scan duration=8:48 minutes, number of animals $=4$. (c) using the solenoid coil: TR/TEeff $=2500 / 552 \mathrm{~ms}, F O V=100 \times 72.2 \times 39.9 \mathrm{~mm}^{3}$, voxel size: $0.30 \times 0.30 \times 0.30 \mathrm{~mm}^{3}$, number of slices acquired $=133$, scan duration $=17: 05$ minutes, with turbo factor $=120$ and refocusing angle $=35^{\circ}$, number of animals $=3$. A high resolution T1weighted magnetization sequence was also performed for 5 animals, parameters were (a) using the human wrist coil: $T R / T E=10.5 / 4.70 \mathrm{~ms}, F O V=135 \times 95 \times 45 \mathrm{~mm}^{3}$, voxel size: $0.34 \times 0.45 \times 0.35 \mathrm{~mm}^{3}$, number of slices acquired=128, scan duration=18:05 minutes, number of animals=2. (b) using the solenoid coil: $T R / T E=11 / 5$, $F O V=100 \times 71.1 \times 39.9$, voxel size: $0.30 \times 0.46 \times 0.30 \mathrm{~mm}^{3}$, number of slices=133, scan duration=14:11 minutes, number of animals $=3$.

Images of each marmoset were reconstructed according to the bi-commissural plane. The lesion volume was measured on native T2 weighted images using semi-automatic segmentation of regions of interest (ROIs) with MRIcron software [25]. In order to create lesion 
overlay maps in a standard space, images and ROIs were rigidly coregistered to a marmoset brain template [26] (the origin of which was changed to the anterior commissure) using SPM12 (Statistical Parametric Mapping, Wellcome department of Cognitive Neurology, London, UK) implemented in Matlab (Mathworks, Inc., http://www.mathworks.com, MA, USA). Lesion overlay maps were then computed in MRIcron. In order to accurately determine the location of individual lesions, we used the on-line digital marmoset atlas ImpecBrain Seg [27]. The marmoset brain template provided with the atlas was rigidly coregistered to the T2weighted scan of each animal, and the transformation matrix was applied to binary atlas compartment ROls (cortical grey matter, subcortical grey matter and white matter) and to atlas probabilistic cortical ROIs (prefrontal, motor, limbic, parietal, insular and occipital). An in-house Matlab script computed the intersection between individual lesion masks and atlas ROIs, allowing us to determine the number of lesioned voxels belonging to the sub-cortical, white matter, and cortical areas. In order to explore the relation between lesion volume and motor impairment, Voxel Lesion Symptom Mapping (VLSM) was performed between lesion volumes at $24 \mathrm{~h}$ and 3 months and dexterity scores from the staircase test using the NPM application in MRIcron.

\section{Histology}

After a minimum of 3 months after surgery, 8 marmosets were administered an anaesthetic (alfaxolone, $9 \mathrm{mg} / \mathrm{kg} \mathrm{IM}$ ) followed by sodium pentobarbital (100 mg/kg intraperitoneal) and intracardially perfused with warm heparin buffer followed by cold $4 \%$ paraformaldehyde (PFA). The brain was extracted from the skull and placed in successive baths of PFA $4 \%$, and $10 \%, 20 \%$ and $30 \%$ sucrose solutions. Brains were cut into $30 \mu \mathrm{m}$ coronal sections on a freezing microtome (Microm HM 450, Microm France). Nissl staining ( $n=8$ ) or LFB (for $2 h 30, n=3$ ) counterstained with cresyl violet were performed according to standard procedure on one in every 12 or 14 sections. Immunostaining of Glial Fibrillary Acid Protein (GFAP) to explore astroglial scarring and of Iba1 to label microglia was performed in four brains. Free-floating sections were permeabilized and blocked for $2 \mathrm{~h}$ in immunohistochemistry (IHC) buffer consisting of $0.1 \%$ Triton $\mathrm{X}-100$ and $4 \%$ goat serum in $0.1 \mathrm{M}$ phosphate buffer (reagents from Sigma). Sections were incubated for $24 \mathrm{~h}$ with primary antibodies (rabbit anti GFAP, Dako \#ZO334, 1:1000 or goat anti Iba1, Abcam \#ab5076, 1:500) diluted in IHC buffer. After washes, 
sections were incubated with respective secondary antibodies diluted in IHC buffer (goat antirabbit AlexaFluor 488 and donkey anti-goat AlexaFluor 568, Invitrogen, both 1:1000) for $2 \mathrm{~h}$ at RT. Nuclei were counterstained with DAPI. Images were acquired with Zeiss confocal LSM710 microscope (Image Core Facility, Centre Physiopathologie Toulouse Purpan). Histology figures were constructed with the ScientiFig plugin under ImageJ [28].

\section{Statistical analysis}

Lesion volumes were compared using non-parametric repeated measures ANOVA (Friedman test) to test for differences between time points. Motor scores at different time points were compared to pre-lesion values to evaluate deficits, and to week 1 values to evaluate recovery, using non-parametric paired Wilcoxon tests corrected for multiple comparisons. Corrected Spearman's correlations were established between lesion volume and motor performance. Results were presented as median (first quartile Q1; third quartile Q3). GraphPad Prism software was used for the statistical analysis. 


\section{RESULTS}

\section{Surgical outcomes}

Surgery was straightforward and only required a small bore-hole $(<1 \mathrm{~mm}$ diameter). Three animals died of respiratory failure during surgery and before malonate injection. Two animals died 2-3 days post-surgery due to the development of extensive oedema that compressed the non-affected hemisphere. We replaced pre-surgery methylprednisolone with Dexamethasone $(2 \mathrm{mg} / \mathrm{kg})$. After the procedure, marmosets were given Dexamethasone twice daily (0.5-1 $\mathrm{mg} / \mathrm{kg}$, IM) for 24-36 hours as needed. No further animals were lost following these changes in medication. All surviving malonate-injected animals ( $n=13,8$ males, 5 females) recovered rapidly and were able to feed without assistance and climb within 1-3 days. Sham animals recovered immediately ( $n=2$, males). The behavioural results of two male animals were excluded from the study due to a lack of sensorimotor impairment, associated with a more prefrontal location of their lesions. This was due to injection at the incorrect stereotaxic coordinates, likely due to a misestimation of the location of the Bregma.

\section{Assessment of upper-limb and global motor function}

\section{Dexterity}

A loss of dexterity in the contralesional upper limb was evidenced, with significantly lower scores after surgery compared to baseline performance in the hill and valley versions of the staircase test $(p=0.012$, week 1 post-injury compared to baseline for both versions of the test, $n=11)$. This deficit persisted 12 weeks after injury $(p=0.012)$. At 48 h post-lesion, animals were still convalescent from surgery and many did not attempt to use the test apparati. Therefore, to assess recovery, scores were compared to the week 1 values. Varying degrees of partial spontaneous recovery were observed starting from week 3 in the valley test $(p=0.034)$ and week 4 in the hill test $(p=0.012)$. Transient heminegligence was observed in the first week, but disappeared in all animals after one or two weeks. This is reflected by a significantly lower score compared to baseline in the ipsilesional upper-limb in the valley staircase $48 \mathrm{~h}(p=0.03)$ and one week ( $p=0.021)$ after injury. Seven animals were tested more than 12 weeks after the injury (data not shown), and their performance did not increase further. Severe chronic deficit of dexterity was observed in the hill (70 \% loss) and valley (62\% loss) staircases for this lesion model (figure 1). The sham animals $(n=2)$ did not display deficits in the staircase test (no 
difference vs baseline values at any time-point, performances in both staircases with the contralesional forelimb were always between 13.5 and 15 points).

\section{Grip strength}

Baseline performance of trained animals was $4.5 \mathrm{~N}$ (Q1: 4.6; Q3: 4.7) for the dominant limb and $4.6 \mathrm{~N}(3.7 ; 5.2)$ for the non-dominant $\operatorname{limb}(\mathrm{n}=9$ animals). Four animals were completely unable to reach towards and pull on the handle with the impaired limb, although careful observation of the marmosets within their home-cage suggested that they did not exhibit a total loss of grip power. A significant decrease in DPT performance at week 12 compared to pre lesion values using the contralesional forelimb was observed in the five animals that were able to use the device $(p=0.04)$. The performance using the contralesional forelimb for these 5 animals was $2.04 \mathrm{~N}(2 ; 3.55), 12$ weeks after injury, corresponding to a $54 \%$ loss of strength. This result suggests a long-lasting loss of the capacity for complex movements that require sequential reaching and prehension with grip strength consequent to malonate lesion (figure 2). However, there was also a significant difference between week 1 and week 12 scores $(p=0.04)$ suggesting partial recovery. A slight decrease in performance using the non-impaired forelimb was observed in the first week following surgery ( $p=0.043$ compared to baseline scores), and could be attributed to convalescence, loss of motivation or damage to uncrossed motor tracts. The sham animals displayed the same level of grip strength as the baseline performance at all time-points (mean baseline performance with the dominant forelimb was $4.8 \pm 0.46 \mathrm{~N}$, mean post-lesion values were between $4.8 \mathrm{~N}$ and $5.1 \mathrm{~N}, \mathrm{n}=2$ ). Grip strength impairment was not as severe in any animal as to prevent climbing the cage mesh using both forelimbs.

\section{Neurological scale}

In the acute and subacute phases, poor global motricity was reflected by lower motor and sensitivity scores of both the contralesional forelimb and hindlimb. The neurological score for the affected side was $5(4 ; 5.5)$ out of 22 (figure 3$) 48$ h post-injury $(n=9)$. As the lesion volumes were large, it is possible that damage extended to the primary motor and somatosensory cortex of the hindlimb. This affected the animals' global posture, and marmosets would often lean on the test apparatus or hold the cage bars to support themselves when standing. Hindlimb weakness recovered in 6 out of 9 animals by week 3 . Motor function and sensitivity 
of the forelimbs remained measurably impaired in 4 out of 9 animals after 12 weeks and was significantly different to baseline values $(p=0.031)$. High recovery of neurological score reflects a relatively moderate degree of sensorimotor disability. The score for the contralesional side 12 weeks after injury was $17(16 ; 21)$, significantly different from the week 1 score $(p=0.031)$. All animals used both forelimbs to climb and to bring food to their mouth by two weeks after surgery, although long-lasting deficits could be detected through careful observation and testing with the staircase and dynamometric pull tests.

\section{Non-invasive evaluation of lesion size and location}

Twenty-four hours and 1 week after malonate injection, T2-weighted MRI revealed extensive vasogenic oedema. From 4 weeks onwards, oedema had resorbed and a cystic cavity had formed at the lesion site. The volume of the lesion, measured on native T2 images, was 340 $(244 ; 432) \mathrm{mm}^{3}(\mathrm{n}=9)$ after 24h, $228(151 ; 304) \mathrm{mm}^{3}(\mathrm{n}=5)$ after 1 week, $125(104 ; 197) \mathrm{mm}^{3}$ $(n=9)$ after 4 weeks and $106(64 ; 153) \mathrm{mm}^{3}(n=9)$ after 12 weeks (figure 4). Comparatively in sham animals injected with DPBS, the mean lesion size due to the needle insertion was $24 \pm 1$ (SD) $\mathrm{mm}^{3}$ after $24 \mathrm{~h}(\mathrm{n}=2), 6 \mathrm{~mm}^{3}$ after 1 week $(\mathrm{n}=1), 4 \pm 2 \mathrm{~mm}^{3}$ after 4 weeks $(\mathrm{n}=2)$ and 0.6 $\mathrm{mm}^{3}$ after 12 weeks $(\mathrm{n}=1)$. Lesion size did not correlate with motor deficits measured using the staircase test, DPT or neurological scale at any time point. No significant results were found with VLSM with dexterity scores. Lesion localization in the chronic phase $(n=12), 12$ weeks post-injury, showed that injury mainly affected cortical grey matter and white matter (table 2). The lesion injured the motor, premotor and prefrontal, and to a lesser degree the limbic and parietal cortices. No correlations were found between motor impairment and lesion volume in the subcortical grey matter and white matter or investigated cortical areas. Finally, to assess remote basal ganglia atrophy, the volume of the putamen was measured by manual delineation on T1 weighted images, excluding two animals in whom the structure was directly injured. Twelve weeks after the lesion, the percent difference between putamen volumes in the healthy and lesioned hemisphere was $27.4(18.3 ; 40.7) \%(n=8)$. This difference may reflect secondary lesions in the putamen.

\begin{tabular}{|l|l|l|}
\cline { 2 - 3 } \multicolumn{1}{c|}{} & Volume of injury $\left(\mathbf{m m}^{\mathbf{3}}\right)$ & Percent of total lesion volume \\
\hline Total lesion & $78.5(50 ; 135.8)$ & 100 \\
\hline Cortical grey matter & $44.5(17.1 ; 76.6)$ & $52.2(45.7 ; 69.3)$ \\
\hline
\end{tabular}




\begin{tabular}{|l|l|l|}
\hline White matter & $27.9(14.5 ; 44.1)$ & $38.4(23.9 ; 43.8)$ \\
\hline Subcortical grey matter & $8.2(2.1 ; 14.9)$ & $7.5(3.8 ; 9.7)$ \\
\hline Frontal/Motor cortex & $21.5(10.7 ; 42.4)$ & $31.8(27.2 ; 38.6)$ \\
\hline Prefrontal cortex & $9.7(4.2 ; 18)$ & $12.4(10.9 ; 13.9)$ \\
\hline Limbic cortex & $1.9(0.5 ; 6)$ & $2(1.3 ; 4.2)$ \\
\hline Parietal cortex & $2.3(0.8 ; 5.9)$ & $3.5(2 ; 4.5)$ \\
\hline
\end{tabular}

Table 2. Lesion volumes in different brain structures and cortical regions twelve weeks after the injury $(n=12)$, expressed as median and quartiles.

\section{Tissue consequences of the lesion}

The ipsilesional internal capsule (IC) appeared narrower, with disorganized fibres after LFB staining (figure 5). Nissl staining revealed an infiltration of cells in the ipsilesional subcortical white matter tracts both near and distant to the lesion site in all lesioned brains analysed $(n=7)$ but not in the sham-lesioned animal $(n=1)$. The infiltration was traceable along the IC, lenticular fasciculus, and down into the cerebral peduncles (figure 5). Interestingly, in these areas, we observed a focal hypo-intensity on T2-weighted MRI images and hyper-intensity on T1-weighted images of sufficient resolution (supplementary figure 1). T1 and T2 abnormalities were observed as early as 1-week post-lesion and persisted thereafter. GFAP and Iba1 immunoreactivity was increased in the ipsilesional IC and lenticular fasciculus relative to the uninjured hemisphere $(n=4)$, and was still observable in the brain of an animal killed 9 month (figure 6) or 1 year after the injury. Remote gliosis and inflammation were observed whether the animals had recovered well or not. Remote from lesion, Iba1-positive cells had an elongated morphology which differed from the round amoeboid morphology of cells found around the lesion. A GFAP positive glial scar surrounded the lesion cavity (data not shown). Injury to the head of the caudate nucleus was also observed, however no pyknotic cells were observed in the striatum nor in the thalamus. 


\section{DISCUSSION}

In this study we demonstrated that stereotaxic injection of mitochondrial toxin malonate causes focal damage that reliably targets the motor cortex, prefrontal cortex and descending motor fibres in the brain of middle-aged marmoset monkeys. Compared to existing models, a key advantage of the malonate model is the low variability in lesion location, associated with consistent moderate to severe fine chronic motor deficits. For the first time in marmosets, we show secondary degeneration and cell infiltration in the white matter associated with a clear MRI signature.

In line with our experience in rats [17], [22], the malonate model was highly reproducible and survivable. However, we report here the death of five marmosets due to respiratory failure during surgery $(n=3)$ and extensive oedema $(n=2)$. Endotracheal intubation may efficiently address the issue of respiratory failure and should be considered in future experiments. Prostration was a sign of cerebral compression caused by oedema, which can be fatal as reported in other non-human primate models of stroke [29]-[31]. In our study, we tackled this complication with high dose of dexamethasone, which improved symptoms drastically and prevented oedema-related mortality. Additionally, the $24 \mathrm{~h} \mathrm{MRI} \mathrm{scan} \mathrm{allowed}$ us to assess oedema and supply additional corticoids if needed. As reported by others [32] [33], severe vasogenic oedema was still present 1 week post-injury but had resolved within a month. One week after surgery, all animals could eat, climb and move with ease and no major disabilities were observed.

The malonate model allowed the assessment of the severity and recovery of motor deficits over at least 12 weeks, using a panel of tests with a strong focus on upper limb function. Here, we present a novel device (DPT), to effectively quantify upper limb grip strength in marmosets with altered motor function, although it requires a relatively long training period. We are the first to report a durable decrease of grip strength in the contralesional upper limb in our model of stroke, unlike previous studies, in which gripping ability was less sensitively assessed [13]. Animals with a DPT score of zero did not exhibit a total loss of upper limb mobilization and grip power but were unable to perform successful synergistic reach-and-pull movements. In addition, we used the staircase test to assess hemineglect (valley version) and forepaw dexterity (hill version) [10], in a modified version that increased difficulty. The valley staircase revealed transient (3-7 days) spatial deficits in 
some animals, shorter than 20 day deficits observed by Marshall after MCAo [34]. Several reasons may explain this discrepancy, such as transient oedema, injury in the left hemisphere for most marmosets, or because the lesion affected the parietal cortex less than MCAo does. Indeed, spatial neglect after stroke in humans is associated with right hemisphere infarction and parietal injury [35]. Some animals exhibited circling behaviour towards the contralesional side, a possible consequence of basal ganglia injury [10], [36], [37]. The neurological scale revealed sensory deficits following malonate-induced injury, which were not taken into account in existing marmoset neurological scales [7]. Our scale is quick and easy to use and does not require videotaping or handling of the animals. Similar to clinical manifestations of ischemic stroke, marmosets exhibited persistent loss of strength and dexterity in the contralesional forepaw, with stable partial recovery after 12 weeks. We noted as also reported in macaque monkeys [38] that some animals exhibited disuse of the impaired limb and would refuse or be reticent to perform the motor tasks. This result could not be explained by motivation since injury only slightly affected the limbic cortex.

Using MRI to non-invasively follow brain injury evolution across time, we show that lesions were reproducible in terms of location, with a small degree of inter-individual variability in lesion volume. Significant tissue loss was observed in the cortex and white matter whereas subcortical damage was far less extensive. Grey matter injury mainly targeted the motor cortex, and secondarily the prefrontal cortex. We did not find any correlation between motor performance and volume of injury in the different brain regions. This is most likely due to the small sample size and/or the fact that the range of injury volumes was small. Prefrontal damage was not linked to motor impairment, possibly because our motor tests were not cognitively challenging. Future studies should investigate potential cognitive deficits, though none were obvious in our study. Only a small percentage of lesion volume affected the parietal cortex, meanwhile sensory deficits were observed in some animals even in the chronic phase. This could be explained by the fact that sensory fibres also end in the primary motor cortex [39].

MRI revealed Wallerian degeneration (WD) in the CST, defined here as degeneration in an anterograde direction following cell body death. In humans, four stages are described: (1) no MRI abnormalities, (2) T2 hypointensity, (3) T2 hyperintensity and (4) tissue atrophy [40]. In our non-human primate model, we observed that the CST was hypointense in T2- 
weighted images, together with a slight shortening of $\mathrm{T} 1$ relaxation time. This signature corresponding to stage 2 WD was observed by our group in stroke patients (supplementary figure 2) but not in rats [22]. Since our T2 sequence included fat suppression, shortening of T2 cannot be interpreted as lipid signal contribution as previously suggested [40]. Rather, it seems consistent with the presence of more restricted water which could be explained by an increase in cell density and gliosis. Moreover, this MRI signature, does not always lead to atrophy in humans [40]. Notably, it was observed in other structures such as the striatum and cortex of stroke patients [41], and was suggested to reflect selective neuronal loss with astroglial and microglial proliferation [41]. Thus, in our model, gliosis likely appeared as early as stage 2 and not in stage 3. To further investigate the altered MRI signal, we performed histopathological analyses in the chronic phase. In all animals screened, Nissl and LFB staining revealed remote damage with hypercellularity and myelin loss in the ipsilesional IC, lenticular fasciculus and cerebral peduncle. Some of the cell nuclei within the infiltration had the irregular, darkly stained morphology, as recently described for microglia [42]. In animals sacrificed 4, 9 and 12 months after the injury, immunostaining showed Iba1-positive cells in the ipsilesional IC. This is consistent with the persistent microglial activation and WD evidenced with positron emission tomography in the subacute and chronic phases of ischemic stroke in humans [43].

To our knowledge, we are the first to describe microglial accumulation in the lenticular fasciculus, a major bundle of efferent pallidothalamic fibres that pass through the posterior limb of the IC. Since the globus pallidus was only directly injured in two animals, remote lenticular fasciculus injury in the other animals likely reflects degeneration of cortico-striatalthalamo-cortical loop axons. Our hypothesis is supported by retrograde tracing studies in marmosets showing that the primary motor cortex receives thalamic input from ventral anterior and ventrolateral nuclei [44], [45] and is consistent with the observed putamen atrophy, as neurons in primary motor cortex project to the putamen [46]. The lesions we observed could also be attributed to damage of other corticopontine fibres such as the reticulospinal tract, known to be involved in hand movement and reaching in humans and macaques [47]. Finally, contrary to our own observations in rats [22] we did not observe pyknotic neurons or cell infiltration in the striatum or thalamus after malonate injection. 
Astrogliosis may also contribute to the MRI abnormalities, since T2 hypointensity has been described around lesions with a thick glial scar in rats [48] and an accumulation of GFAP cells was found in T2 hypointense grey matter lesions after focal stroke in neonatal rats [49]. As expected, we found a thin GFAP positive thin glial scar surrounding the lesion cavity which was not detectable by T2 weighted MRI. Interestingly, at 4, 8 and 12 months after malonate injection, remote from the lesion, we observed an accumulation of GFAP positive cells which seemed aligned along the initial direction of the CST and lenticular fasciculus fibres.

Future studies should help elucidate the significance of chronic hypercellularity in relation to motor function. Indeed, hypercellularity and gliosis may reflect persistent detrimental inflammation [50]. However, they also participate in tissue remodelling through clearance of debris and axonal growth inhibitors, induction of axonal regrowth, and promotion of neuro- and oligodendrogenesis [50]-[55]. Mechanisms may however be difficult to elucidate given the wide spectrum of microglia and astrocyte phenotypes with overlapping gene expression and functions [56], also seen in humans [57]. Well-defined validated markers of anti- and pro-inflammatory states are lacking [57], and are especially rare in newer experimental animals such as marmosets [55], [56].

In conclusion, stereotaxic injection of the mitochondrial toxin malonate results in a focal brain lesion with anatomical and functional consequences comparable to ischemic stroke. Two key advantages of our model are (i) reproducible lesion location and (ii) the presence of measurable long-lasting moderate motor deficits. To our knowledge, we are the first to report persistent hypercellularity remote from the lesion in the CST and subcortical motor loops with an associated MRI signature, after cortical ischemic injury. The malonateinduced cortical injury model may therefore be successfully used in marmosets to assess therapeutic strategies for motor recovery, particularly in the chronic phase after stroke.

\section{ACKNOWLEDGMENTS}

We thank Benjamin Plas for assistance and Robert Darmana for Dynamometric Pull Test design. We thank Florence Capilla of the Experimental Histopathology Platform of INSERM US06/CREFRE for Luxol Fast Blue staining. We thank the Inserm/UPS UMR1214 Technical Platform for their help in setting up and acquisition of the MR images, and the Image Core Facility of the Centre Physiopathologie Toulouse Purpan for confocal microscopy. We thank Animalliance staff Celine Monzali, Marie Mercier, Celine Martinez and Christelle Laravine for care of the marmosets. The study was funded by the Société Fédérative de Recherche (SFR), 
Toulouse Mind and Brain Institute (Institut Des Sciences Du Cerveau De Toulouse), Région Midi-Pyrénées and Fondation Institut de France NRJ.

\section{CONFLICT OF INTEREST STATEMENT}

Nothing to disclose

The data that support the findings of this study are available from the corresponding author upon reasonable request.

\section{Author Contribution}

Study design, experimental work, analysis and interpretation of the data, draft and editing of the manuscript [Alice Le Friec, Franck Desmoulin]; experimental work, draft and editing of the manuscript [Carla Cirillo]; MRI experts, experimental work and analysis and interpretation of the data [Franck Desmoulin, Florence Remy]; experimental work [Boris Demain, Carole Davoust, Tanguy Duval, Lorenne Robert]; study conception and design, analysis and interpretation of the data, draft and editing of the manuscript, obtaining funding [Isabelle Loubinoux].

\section{Ethical Approval}

All animals were maintained and treated according to Council of the European Communities guidelines (EU Directive 2010/63). Our protocol was approved by the Direction départementale de la Protection des Populations de la Haute-Garonne and the Comité d'éthique pour l'expérimentation animale Midi-Pyrénées (authorization $n^{\circ} 31125507$ and $n^{\circ}$ B3155501). 


\section{References}

[1] J. Bogousslavsky, G. Van Melle, and F. Regli, "The Lausanne Stroke Registry: analysis of 1,000 consecutive patients with first stroke.," Stroke, vol. 19, no. 9, pp. 1083-1092, Sep. 1988.

[2] D. Kaya, A. Dincer, F. Arman, N. Bakirci, C. Erzen, and M. N. Pamir, "Ischemic Involvement of the Primary Motor Cortex is a Prognostic Factor in Acute Stroke," International Journal of Stroke, vol. 10, no. 8, pp. 1277-1283, Dec. 2015.

[3] C. M. Stinear, P. A. Barber, P. R. Smale, J. P. Coxon, M. K. Fleming, and W. D. Byblow, "Functional potential in chronic stroke patients depends on corticospinal tract integrity," Brain, vol. 130, no. 1, pp. 170-180, Nov. 2006.

[4] A. Ganesh et al., "Time Course of Evolution of Disability and Cause-Specific Mortality After Ischemic Stroke: Implications for Trial Design," Journal of the American Heart Association, vol. 6, no. 6, Nov. 2017.

[5] V. Kuptniratsaikul, A. Kovindha, S. Suethanapornkul, P. Massakulpan, W. Permsirivanich, and P. S. Kuptniratsaikul, "Motor recovery of stroke patients after rehabilitation: one-year follow-up study," International Journal of Neuroscience, vol. 127, no. 1, pp. 37-43, Jan. 2017.

[6] M. Fisher et al., "Update of the Stroke Therapy Academic Industry Roundtable Preclinical Recommendations," Stroke, vol. 40, no. 6, pp. 2244-2250, Jun. 2009.

[7] R. Le Gal, M. Bernaudin, J. Toutain, and O. Touzani, "Assessment of behavioural deficits following ischaemic stroke in the marmoset," Behavioural Brain Research, Jul. 2018;352:151-160.

[8] J. W. B. Marshall, A. J. Cross, D. M. Jackson, A. R. Green, H. F. Baker, and R. M. Ridley, "Clomethiazole protects against hemineglect in a primate model of stroke," Brain Research Bulletin, vol. 52, no. 1, pp. 21-29, May 2000.

[9] J. W. B. Marshall, R. M. Cummings, L. J. Bowes, R. M. Ridley, and A. R. Green, "Functional and Histological Evidence for the Protective Effect of NXY-059 in a Primate Model of Stroke When Given 4 Hours After Occlusion," Stroke, vol. 34, no. 9, pp. 2228-2233, Sep. 2003.

[10] J. W. B. Marshall and R. M. Ridley, "Assessment of Functional Impairment Following Permanent Middle Cerebral Artery Occlusion in a Non-Human Primate Species," Neurodegeneration, vol. 5, no. 3, pp. 275-286, Sep. 1996.

[11] J. Marshall, "Serial MRI, functional recovery, and long-term infarct maturation in a non-human primate model of stroke," Brain Research Bulletin, vol. 61, no. 6, pp. 577-585, Oct. 2003.

[12] D. Virley et al., "A New Primate Model of Focal Stroke: Endothelin-1-Induced Middle Cerebral Artery Occlusion and Reperfusion in the Common Marmoset," Journal of Cerebral Blood Flow \& Metabolism, vol. 24, no. 1, pp. 24-41, Jan. 2004.

[13] T. Freret et al., "Intraluminal thread model of focal stroke in the non-human primate," Journal of Cerebral Blood Flow \& Metabolism, vol. 28, no. 4, pp. 786-796, 2008.

[14] E. Bihel et al., "Permanent or transient chronic ischemic stroke in the non-human primate: behavioral, neuroimaging, histological, and immunohistochemical investigations," Journal of Cerebral Blood Flow \& Metabolism, vol. 30, no. 2, pp. 273-285, 2010.

[15] S. Ikeda, K. Harada, A. Ohwatashi, Y. Kamikawa, A. Yoshida, and K. Kawahira, "A New Non-Human Primate Model of Photochemically Induced Cerebral Infarction," PLOS ONE, vol. 8, no. 3, p. e60037, Mar. 2013.

[16] L. Barros-Miñones et al., "Modulation of the ASK1-MKK3/6-p38/MAPK signalling pathway mediates sildenafil protection against chemical hypoxia caused by malonate: Sildenafil and chemical hypoxia," British Journal of Pharmacology, vol. 168, no. 8, pp. 1820-1834, Apr. 2013.

[17] C. Cirillo et al., "Focal Malonate Injection Into the Internal Capsule of Rats as a Model of Lacunar Stroke," Frontiers in Neurology, vol. 9, Dec. 2018;9:1072.

[18] C. Davoust et al., "Regenerative potential of primary adult human neural stem cells on micropatterned bioimplants boosts motor recovery.," Stem Cells Research \& Therapy, 2017;8(1):253. 
[19] J. G. Greene, R. H. P. Porter, R. V. Eller, and J.-T. Greenamyre, "Inhibition of Succinate Dehydrogenase by Malonic Acid Produces an 'Excitotoxic' Lesion in Rat Striatum," Journal of Neurochemistry, vol. 61, no. 3, pp. 1151-1154, Sep. 1993.

[20] C. Morland, K. A. Boldingh, E. G. Iversen, and B. Hassel, "Valproate is Neuroprotective against Malonate Toxicity in Rat Striatum: An Association with Augmentation of High-Affinity Glutamate Uptake," Journal of Cerebral Blood Flow \& Metabolism, vol. 24, no. 11, pp. 1226-1234, Nov. 2004.

[21] L. Vaysse, A. Beduer, J. C. Sol, C. Vieu, and I. Loubinoux, "Micropatterned bioimplant with guided neuronal cells to promote tissue reconstruction and improve functional recovery after primary motor cortex insult," Biomaterials, vol. 58, pp. 46-53, Jul. 2015.

[22] L. Vaysse et al., "Strength and fine dexterity recovery profiles after a primary motor cortex insult and effect of a neuronal cell graft.," Behavioral Neuroscience, vol. 129, no. 4, pp. 423-434, 2015.

[23] S. Yuasa, S. Kohsaka, and K. Nakamura, Stereotaxic atlas of the marmoset brain: with immunohistochemical architecture and MR images. National Institute of Neuroscience, National Center of Neurology and Psychiatry, Japan., 2010.

[24] K. Kitamura et al., "Human Hepatocyte Growth Factor Promotes Functional Recovery in Primates after Spinal Cord Injury," PLOS ONE, vol. 6, no. 11, p. e27706, Nov. 2011.

[25] C. Rorden and M. Brett, "Stereotaxic Display of Brain Lesions," Behavioural Neurology, vol. 12, no. 4, pp. 191-200, 2000.

[26] K. Hikishima et al., "Population-averaged standard template brain atlas for the common marmoset (Callithrix jacchus)," Neurolmage, vol. 54, no. 4, pp. 2741-2749, Feb. 2011.

[27] L. Risser et al., "In vivo localization of cortical areas using a 3D computerized atlas of the marmoset brain," Brain Struct Funct, Apr. 2019;224(5):1957-1969.

[28] B. Aigouy and V. Mirouse, "ScientiFig: a tool to build publication-ready scientific figures," Nature Methods, vol. 10, no. 11, pp. 1048-1048, Nov. 2013.

[29] H. E. D'Arceuil, M. Duggan, J. He, J. Pryor, and A. Crespigny, "Middle cerebral artery occlusion in Macaca fascicularis: acute and chronic stroke evolution," J Med Primatol, vol. 35, no. 2, pp. 7886, Apr. 2006.

[30] G. Ramirez-Garcia, K. A. Harrison, J. Fernandez-Ruiz, J. Y. Nashed, and D. J. Cook, "Stroke Longitudinal Volumetric Measures Correlate with the Behavioral Score in Non-Human Primates," Neuroscience, vol. 397, pp. 41-55, Jan. 2019.

[31] D. Wu, A. Chandra, J. Chen, Y. Ding, and X. Ji, "Endovascular Ischemic Stroke Models in Nonhuman Primates," Neurotherapeutics, vol. 15, no. 1, pp. 146-155, Jan. 2018.

[32] P. Dai et al., "A pilot study on transient ischemic stroke induced with endothelin-1 in the rhesus monkeys," Sci Rep, vol. 7, no. 1, p. 45097, Dec. 2017.

[33] L. Teo and J. A. Bourne, "A Reproducible and Translatable Model of Focal Ischemia in the Visual Cortex of Infant and Adult Marmoset Monkeys: Focal Injury in Marmoset Primary Visual Cortex," Brain Pathology, vol. 24, no. 5, pp. 459-474, Sep. 2014.

[34] J. W. Marshall and R. M. Ridley, "Assessment of cognitive and motor deficits in a marmoset model of stroke," ILAR journal, vol. 44, no. 2, pp. 153-160, 2003.

[35] A. Bowen, K. McKenna, and R. C. Tallis, "Reasons for Variability in the Reported Rate of Occurrence of Unilateral Spatial Neglect After Stroke," Stroke, vol. 30, no. 6, pp. 1196-1202, Jun. 1999.

[36] S. Puentes, T. Kaido, T. Hanakawa, N. Ichinohe, T. Otsuki, and K. Seki, "Internal capsule stroke in the common marmoset," Neuroscience, vol. 284, pp. 400-411, Jan. 2015.

[37] A. L. Kendall, "The influence of excitotoxic basal ganglia lesions on motor performance in the common marmoset," Brain, vol. 123, no. 7, pp. 1442-1458, Jul. 2000.

[38] W. G. Darling et al., "Sensorimotor cortex injury effects on recovery of contralesional dexterous movements in Macaca mulatta," Experimental Neurology, vol. 281, pp. 37-52, Jul. 2016.

[39] J. C. Rothwell, Control of Human Voluntary Movement. Springer US, 1987.

[40] M. J. Kuhn, D. J. Mikulis, D. M. Ayoub, B. E. Kosofsky, K. R. Davis, and J. M. Taveras, "Wallerian degeneration after cerebral infarction: evaluation with sequential MR imaging," Radiology, vol. 172, no. 1, pp. 179-182, Jul. 1989. 
[41] M. Fujioka, T. Taoka, K.-I. Hiramatsu, S. Sakaguchi, and T. Sakaki, "Delayed Ischemic Hyperintensity on T1-Weighted MRI in the Caudoputamen and Cerebral Cortex of Humans After Spectacular Shrinking Deficit," Stroke, vol. 30, no. 5, pp. 1038-1042, May 1999.

[42] M. Á. García-Cabezas, Y. J. John, H. Barbas, and B. Zikopoulos, "Distinction of Neurons, Glia and Endothelial Cells in the Cerebral Cortex: An Algorithm Based on Cytological Features," Frontiers in Neuroanatomy, vol. 10, Nov. 2016;10:107.

[43] A. Thiel and W.-D. Heiss, "Imaging of Microglia Activation in Stroke," Stroke, vol. 42, no. 2, pp. 507-512, Feb. 2011.

[44] K. J. Huffman and L. Krubitzer, "Thalamo-cortical connections of areas 3a and M1 in marmoset monkeys," J. Comp. Neurol., vol. 435, no. 3, pp. 291-310, Jul. 2001.

[45] K. J. Burman, S. Bakola, K. E. Richardson, D. H. Reser, and M. G. P. Rosa, "Patterns of cortical input to the primary motor area in the marmoset monkey: Afferent connections of the motor cortex," J. Comp. Neurol., vol. 522, no. 4, pp. 811-843, Mar. 2014.

[46] B. Demain et al., "Corticospinal Tract Tracing in the Marmoset with a Clinical Whole-Body 3T Scanner Using Manganese-Enhanced MRI.," PLOS ONE, p. 10(9):e0138308, 2015.

[47] S. N. Baker, "The primate reticulospinal tract, hand function and functional recovery: Primate reticulospinal tract," The Journal of Physiology, vol. 589, no. 23, pp. 5603-5612, Dec. 2011.

[48] H. Li et al., "Histological, cellular and behavioral assessments of stroke outcomes after photothrombosis-induced ischemia in adult mice," BMC Neurosci, vol. 15, no. 1, p. 58, 2014.

[49] C. T. van Velthoven et al., "Mesenchymal stem cells attenuate MRI-identifiable injury, protect white matter, and improve long-term functional outcomes after neonatal focal stroke in rats: Effect of MSC Treatment After Stroke," Journal of Neuroscience Research, vol. 95, no. 5, pp. 1225-1236, May 2017.

[50] Y. Ma, J. Wang, Y. Wang, and G.-Y. Yang, "The biphasic function of microglia in ischemic stroke," Progress in Neurobiology, vol. 157, pp. 247-272, Oct. 2017.

[51] P. Thored et al., "Long-term accumulation of microglia with proneurogenic phenotype concomitant with persistent neurogenesis in adult subventricular zone after stroke," Glia, vol. 57, no. 8, pp. 835-849, Jun. 2009.

[52] H. Neumann, M. R. Kotter, and R. J. M. Franklin, "Debris clearance by microglia: an essential link between degeneration and regeneration," Brain, vol. 132, no. 2, pp. 288-295, Jun. 2008.

[53] J. L. Zamanian et al., "Genomic Analysis of Reactive Astrogliosis," Journal of Neuroscience, vol. 32, no. 18, pp. 6391-6410, May 2012.

[54] M. A. Anderson et al., "Astrocyte scar formation aids central nervous system axon regeneration," Nature, vol. 532, no. 7598, pp. 195-200, Apr. 2016.

[55] H. L. Walter et al., "In vivo analysis of neuroinflammation in the late chronic phase after experimental stroke," Neuroscience, vol. 292, pp. 71-80, Apr. 2015.

[56] K. A. Jones et al., "Peripheral immune cells infiltrate into sites of secondary neurodegeneration after ischemic stroke," Brain, Behavior, and Immunity, vol. 67, pp. 299-307, Jan. 2018.

[57] T. Zrzavy et al., "Dominant role of microglial and macrophage innate immune responses in human ischemic infarcts: Inflammation in Ischemic Lesions," Brain Pathology, vol. 28, no. 6, pp. 791805, Nov. 2018. 


\section{FIGURE LEGENDS}

Fig 1. Dexterity test. Performance in the staircase $(n=11)$ of marmosets after malonate injection. Hill staircase: The curve of the contralesional forepaw (right) shows that lesioned marmosets had a complete drop to zero in performance starting at $48 \mathrm{~h}$ post-lesion, compared to the ipsilesional forepaw (left). Partial recovery of dexterity of the contralesional forepaw was observed starting at week 4 post-lesion. Almost total recovery of dexterity of the ipsilesional forepaw was observed starting at week 2 post-lesion. Valley staircase: The curves of the contralesional (right) and ipsilesional (left) forepaw show that lesioned marmosets had a complete drop to zero in performance starting at $48 \mathrm{~h}$ post-lesion. Partial recovery of dexterity of the contralesional forepaw was observed starting at week 3 post-lesion. Almost total recovery of dexterity of the ipsilesional forepaw was observed starting at week 2 postlesion. Data in the graphs are displayed as median values and interquartile range. ${ }^{*} p<0.05$ compared to pre-lesion values indicating deficits, $\# p<0.05$ compared to week 1 values indicating recovery. Paired non-parametric Wilcoxon test with Benjamini (FDR) correction for multiple comparisons

Fig 2. Strength test. Left: photograph of a marmoset performing the Dynamometric Pull Test (DPT), in which the animal must reach through the opening of the apparatus, then grasp and pull on a handle attached to a dynamometer to release small treats. Right: grip strength in Newtons before and after lesion induction $(n=5)$. Data in the graphs are displayed as median with interquartile range. Paired non-parametric Wilcoxon test shows a significant difference between week 12 and baseline values, revealing a long lasting deficit $\left({ }^{*} p<0.05\right)$. There was also a significant difference between week 12 and week 1 values, showing that some degree of spontaneous recovery occurs $(\# p<0.05)$

Fig 3. Neurological scale (values between 0 and 22) representing the severity of sensorimotor deficits in marmosets $(n=9)$ after malonate injection. Paired non-parametric Wilcoxon test shows that animals did exhibit some degree of motor recovery, since their score was significantly higher twelve weeks compared to one week after surgery $(\# p<0.05)$. A significant difference between week 12 and baseline values revealed a long lasting deficit ( $\left.{ }^{*} p<0.05\right)$. Data in the graph are displayed as median values and interquartile range

Fig 4. MRI lesion location. Lesion overlay maps created in MRIcron ( $n=9$ at $24 \mathrm{~h}$, week 4 and week $12, n=5$ at week 1 post-lesion) show a reproducible lesion location. Lesion volumes of interest were drawn on the T2w images, which were then coregistered to a marmoset brain template. Warm colours show areas that were lesioned in most or all of the animals, and cool colours show areas that were lesioned in fewer animals. The slice numbers represent the approximate distance in $\mathrm{mm}$ relative to the Bregma

Fig 5. Wallerian degeneration. MRI and histological analyses 4 months post-lesion reveal remote injury in marmoset brain. T2-weighted (a, b, e, f) and T1-weighted (c, d, g, h) MRI images show an abnormal signal that is $\mathrm{T} 2$ hypointense and $\mathrm{T} 1$ hyperintense along the injured corticospinal tract $(\mathrm{e}, \mathrm{g})$. Alterations are visible in the internal capsule (arrowheads in a, c) and in pallidothalamic fibres (lenticular fasciculus) remote to the lesion site (arrowheads in $b, d$ ) in comparison to the healthy hemisphere (f, h). Semithin $30 \mu \mathrm{m}$ Nissl-stained whole section (i, 
j) shows remote cell infiltration in the injured internal capsule (arrowhead in i, k) and lenticular fasciculus (arrowhead in j, m). Note the irregular, microglia-resembling morphology of the nuclei within the ipsilesional white matter tracts (see insets of $\mathrm{k} \& \mathrm{~m}$ ). The healthy hemisphere is shown in $L$ and $N$. Semithin $30 \mu \mathrm{m}$ Luxol Fast Blue counterstained with cresyl-violet whole sections $(o, p)$ reveal myelin loss in the areas of cell infiltration $(q, s)$ compared to the noninjured hemisphere $(r, t)$ in a different animal 11 months after injury. Distance from Bregma is approximately $+3.9 \mathrm{~mm}(\mathrm{a}, \mathrm{c}, \mathrm{i})$ and $+2.9 \mathrm{~mm}(\mathrm{~b}, \mathrm{~d}, \mathrm{j})$. Scale bars: $5000 \mu \mathrm{m}(\mathrm{i}, \mathrm{j}, \mathrm{o}, \mathrm{p}), 100 \mu \mathrm{m}(\mathrm{k}$, $\mathrm{l}, \mathrm{m}, \mathrm{n}), 500 \mu \mathrm{m}(\mathrm{q}, \mathrm{r}, \mathrm{s}, \mathrm{t})$.

Fig 6. White matter gliosis. Histological analysis 9 months post-lesion reveals chronic remote white matter inflammation. GFAP (c-h) and Iba1 (e-j) immunolabelling overlaps with the cell infiltration observed with Nissl staining in the ipsilesional internal capsule (a, approximately $+3.9 \mathrm{~mm}$ from Bregma) and lenticular fasciculus (b, approximately $+2.9 \mathrm{~mm}$ from Bregma). The injured hemisphere is presented in a, c, e (internal capsule) and b, g, i (lenticular fasciculus). The healthy hemisphere is presented in $d, f, h$ and $j$. Scale bar $=5000 \mu \mathrm{m}(a, b)$ and $100 \mu \mathrm{m}(c-$ j)

Supplementary Figure 1. MRI optimization. T2 and T1 weighted acquisitions 24h, 1 week and 12 weeks post-lesion show a clear improvement in signal to noise ratio and resolution obtained using a solenoid coil. The acquisitions using the knee, wrist and solenoid coil correspond to three different marmosets. Using the wrist coil, later confirmed using the solenoid coil, we observed a white matter alteration that was T2 hypointense and T1 hyperintense in the subacute and chronic phase

Supplementary Figure 2. Human wallerian degeneration. T2-weighted-FLAIR and T1weighted axial MR images of a patient with motor deficits 4 weeks after ischemic stroke presenting T2 hypo- and T1 hyperintensities in the internal capsule and cerebral peduncle, representing the described signature for stage 2 Wallerian degeneration 
HILL STAIRCASE

Ipsilesional forelimb

Contralesional forelimb
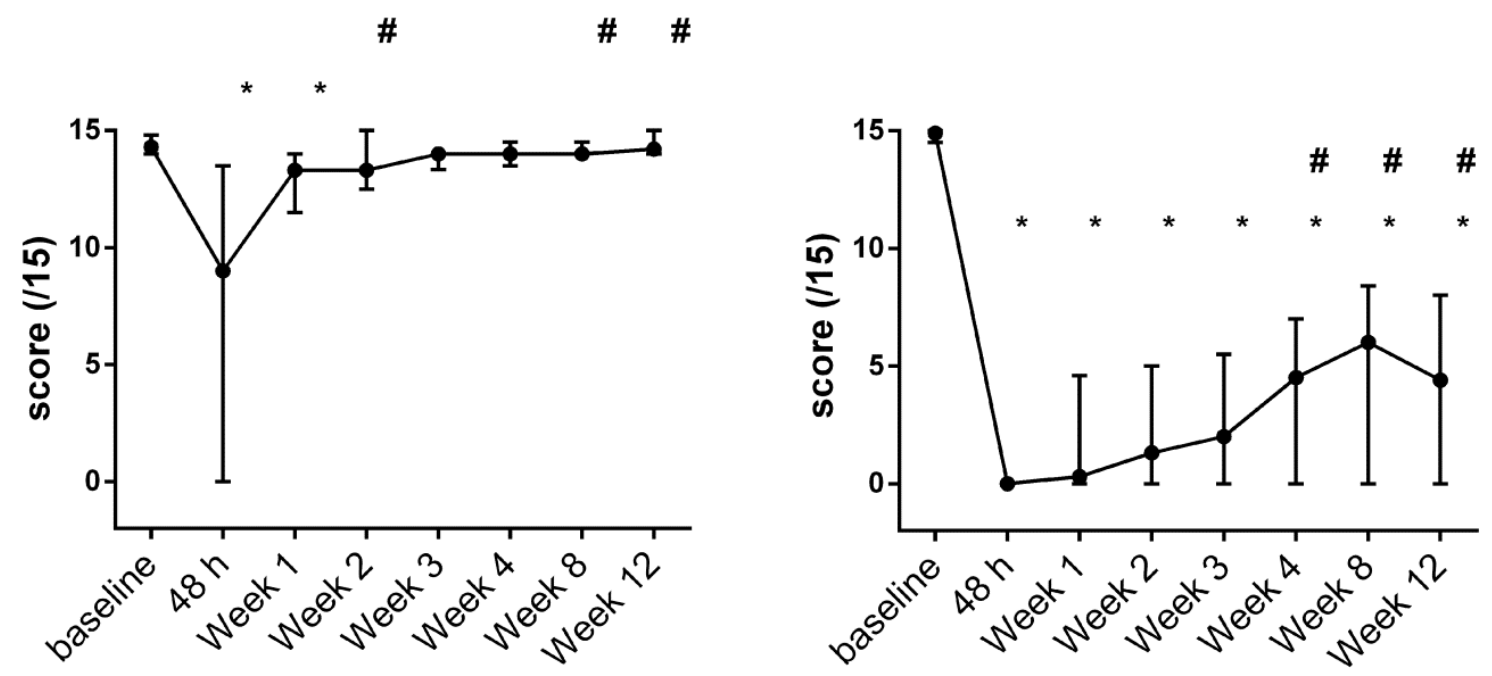

VALLEY STAIRCASE

Ipsilesional forelimb

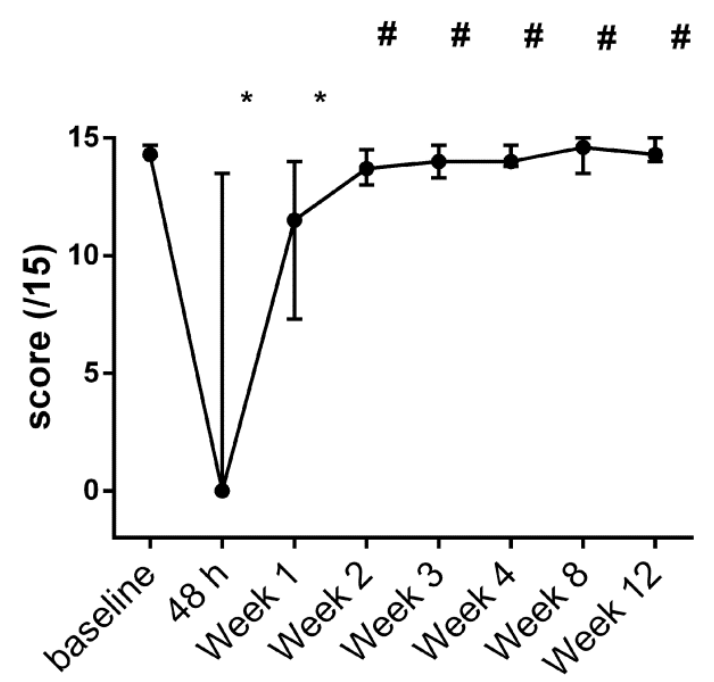

Contralesional forelimb

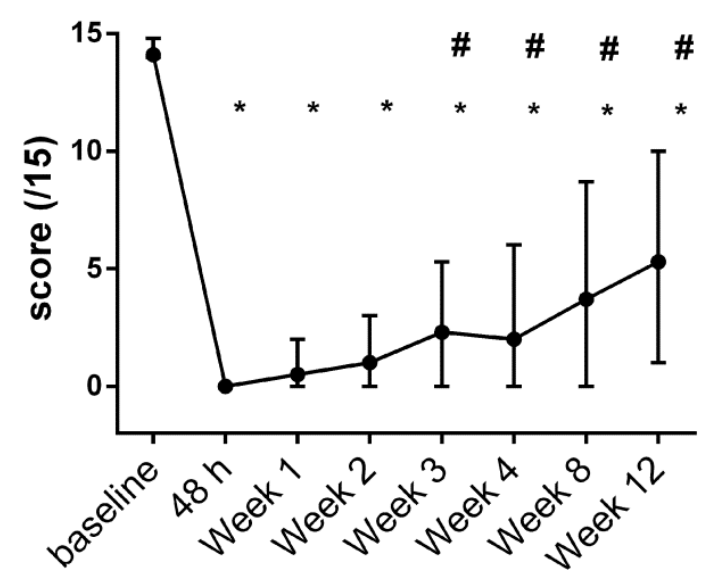

Figure 1 

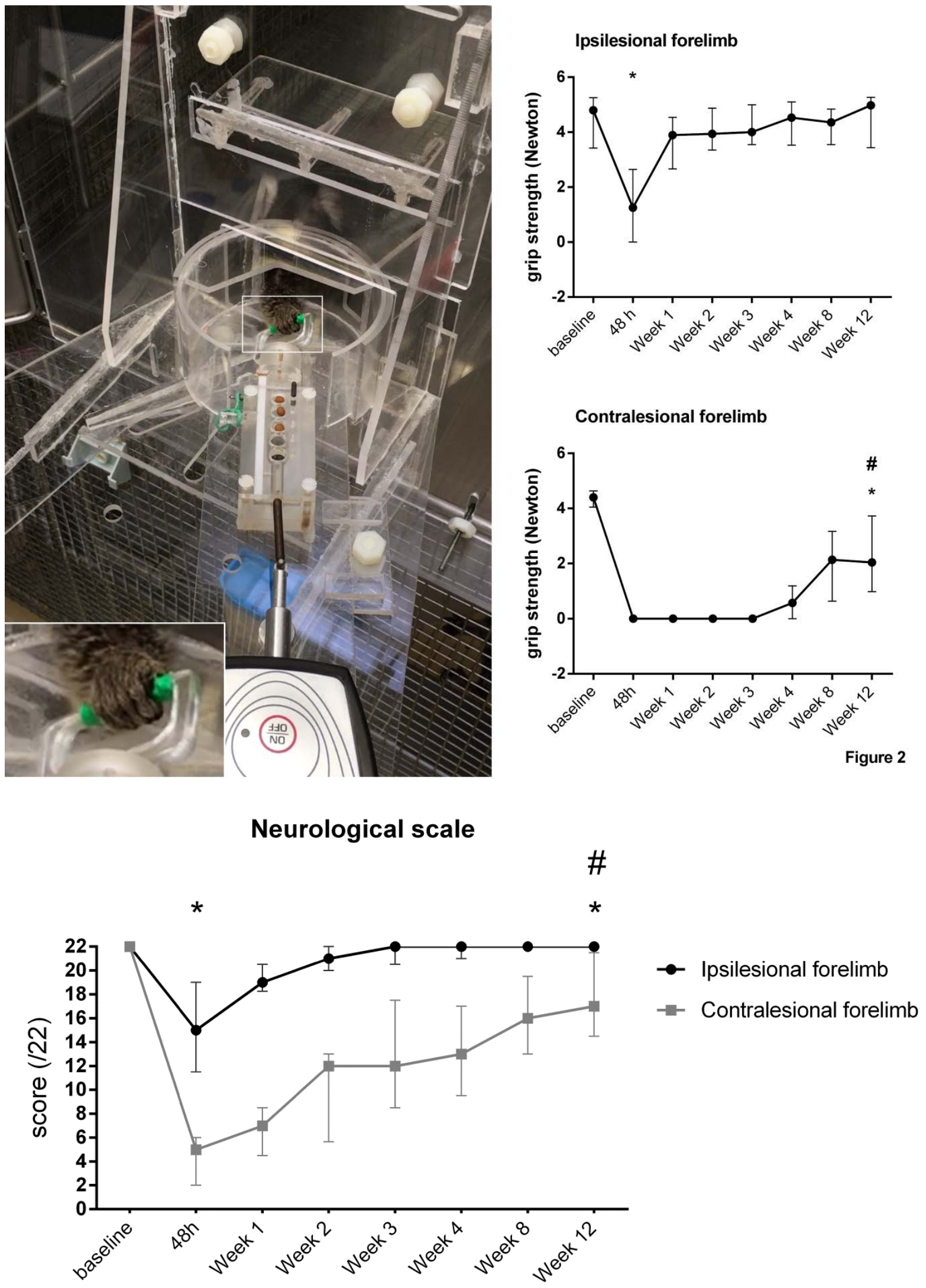

Figure 3 


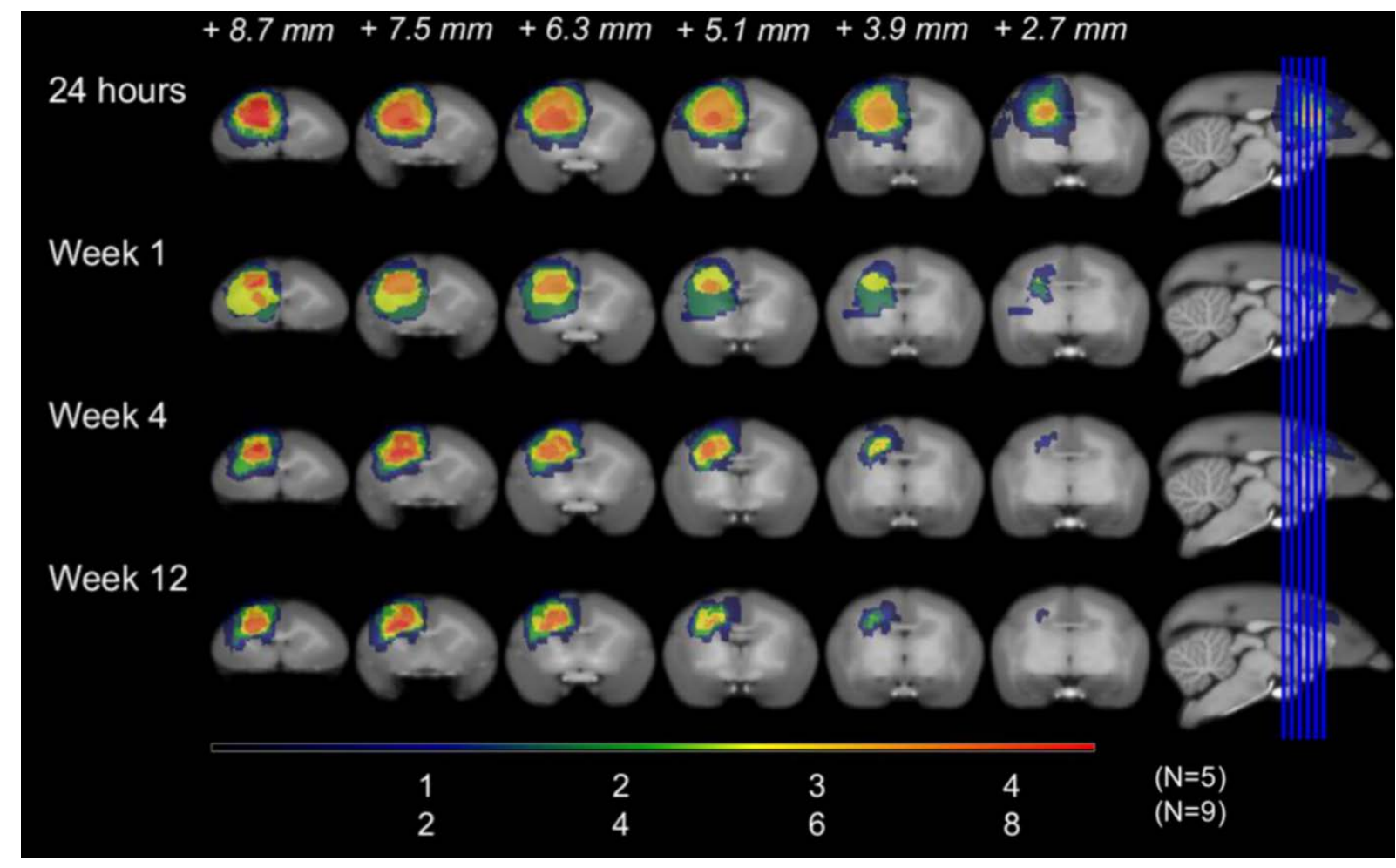

Figure 4 

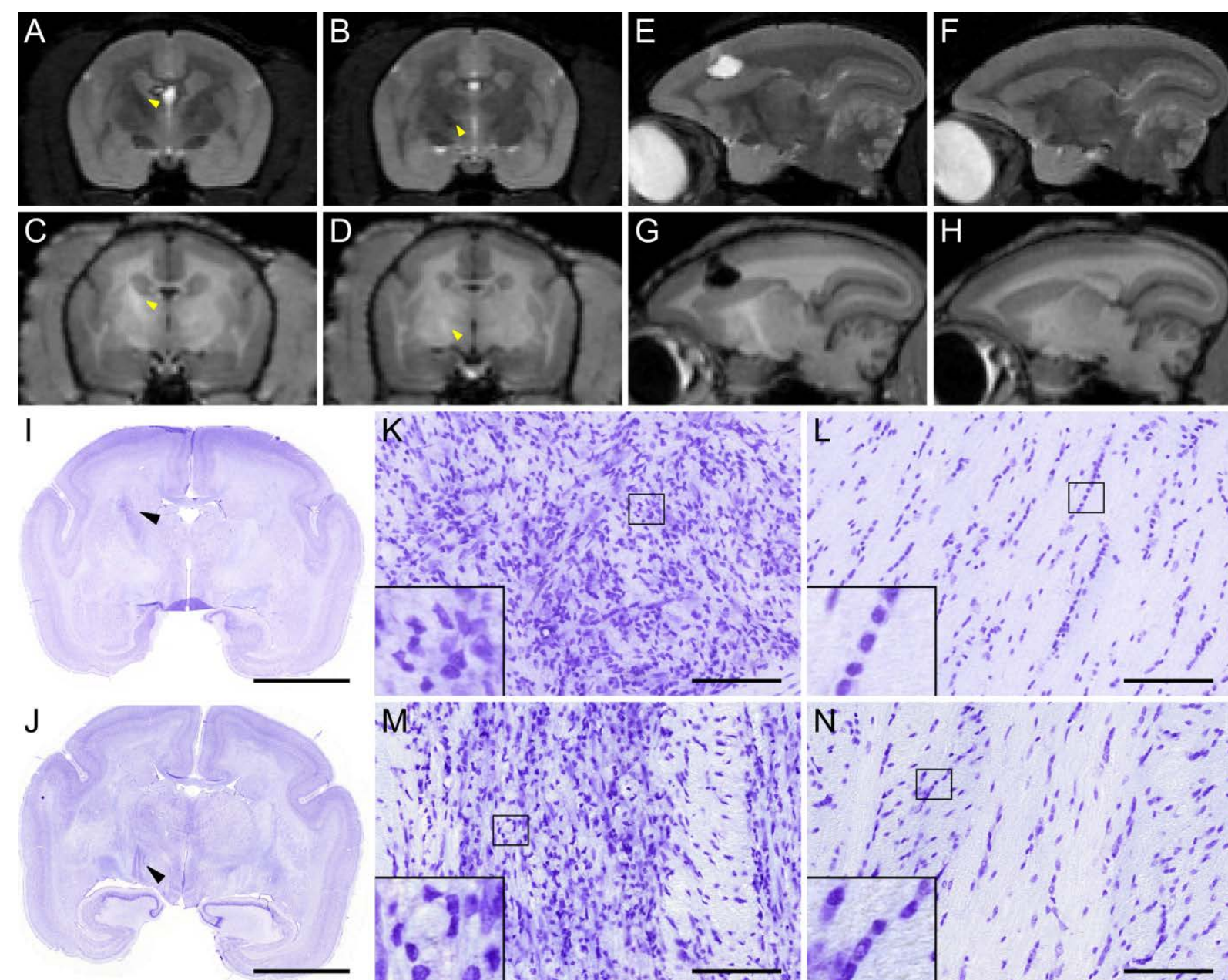

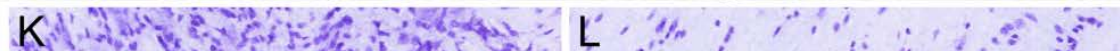

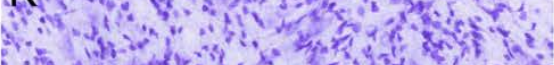
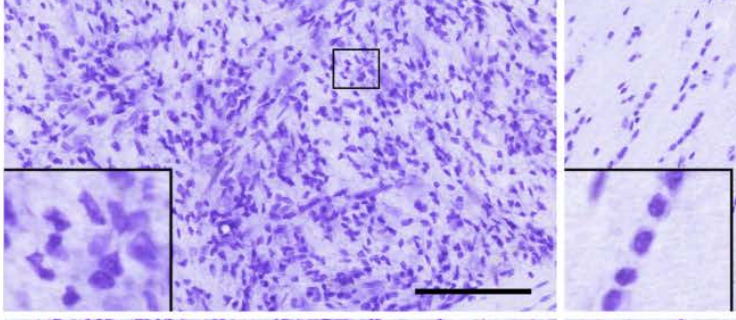

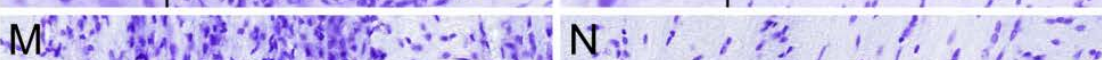

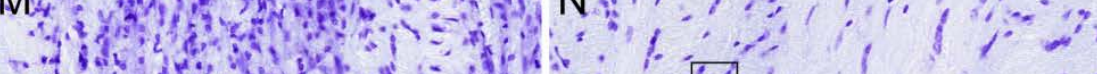

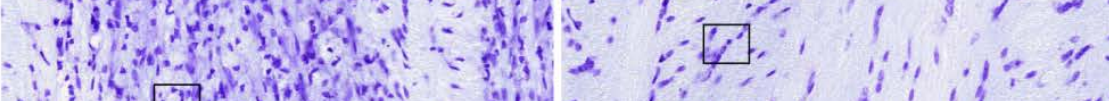
s.

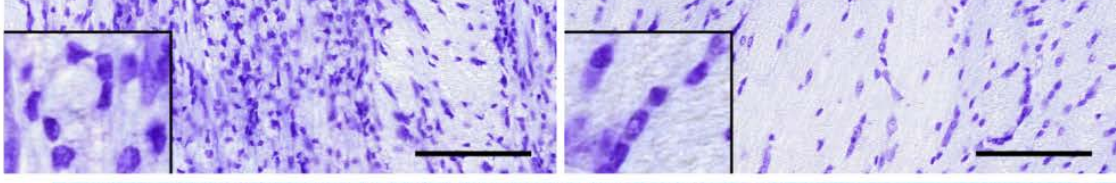

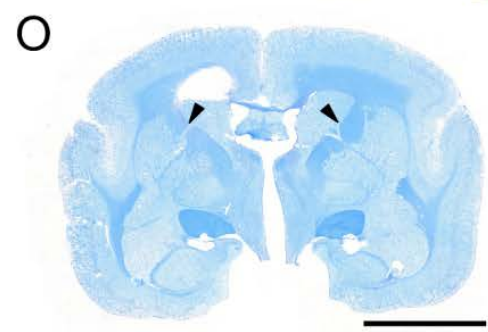

Q

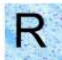

$\mathrm{P}$

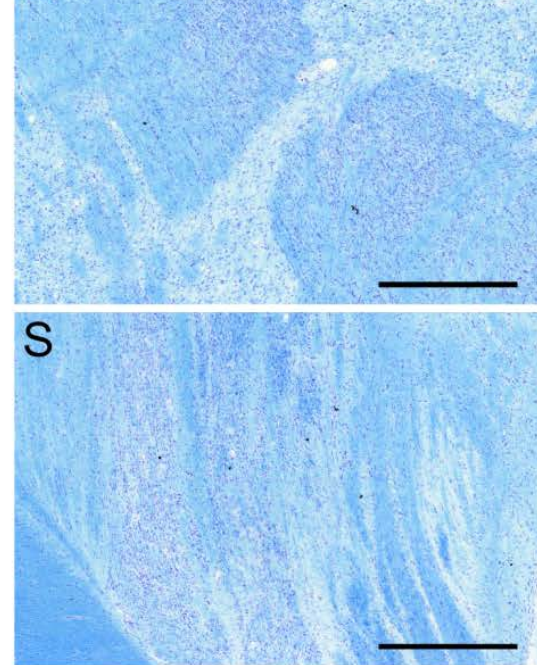

$\mathrm{R}$

Figure 5 


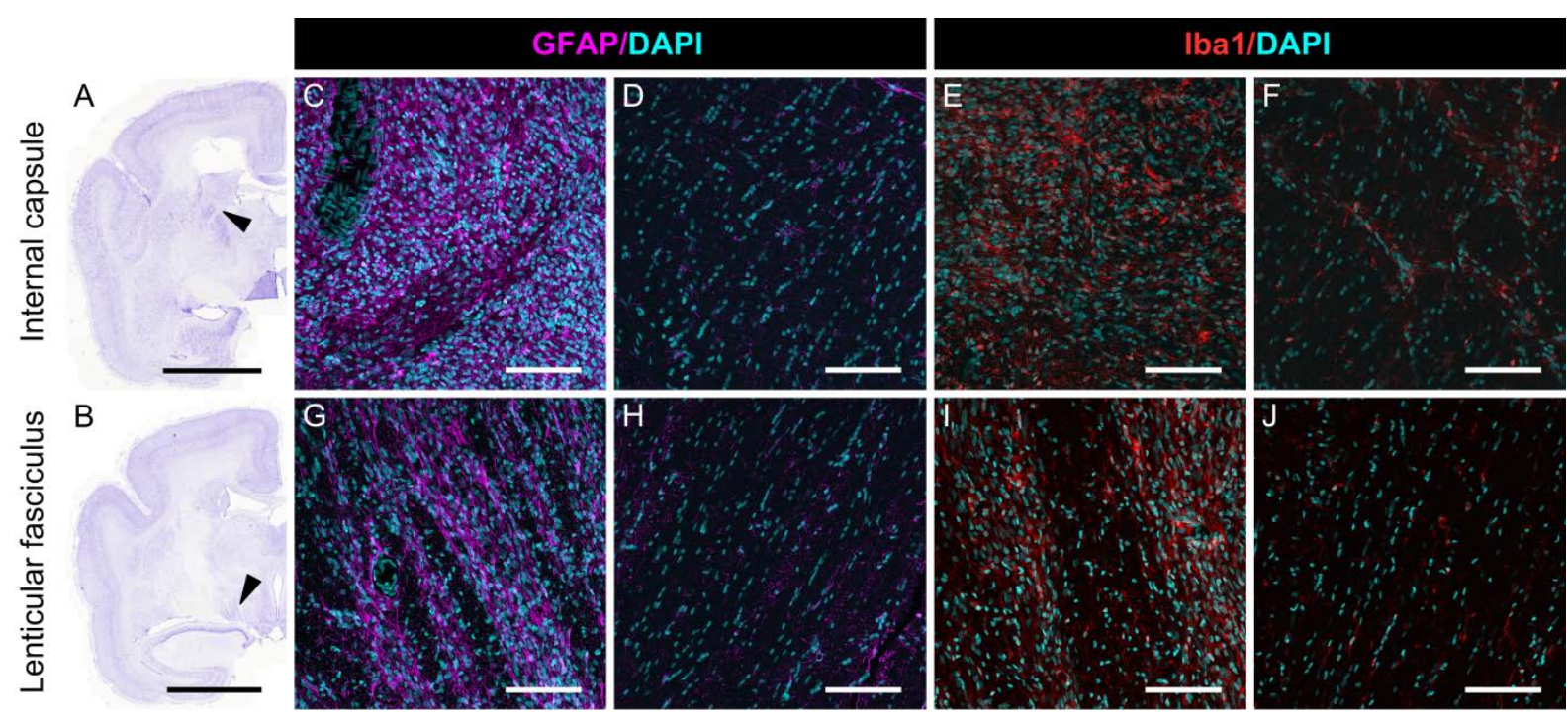

Figure 6
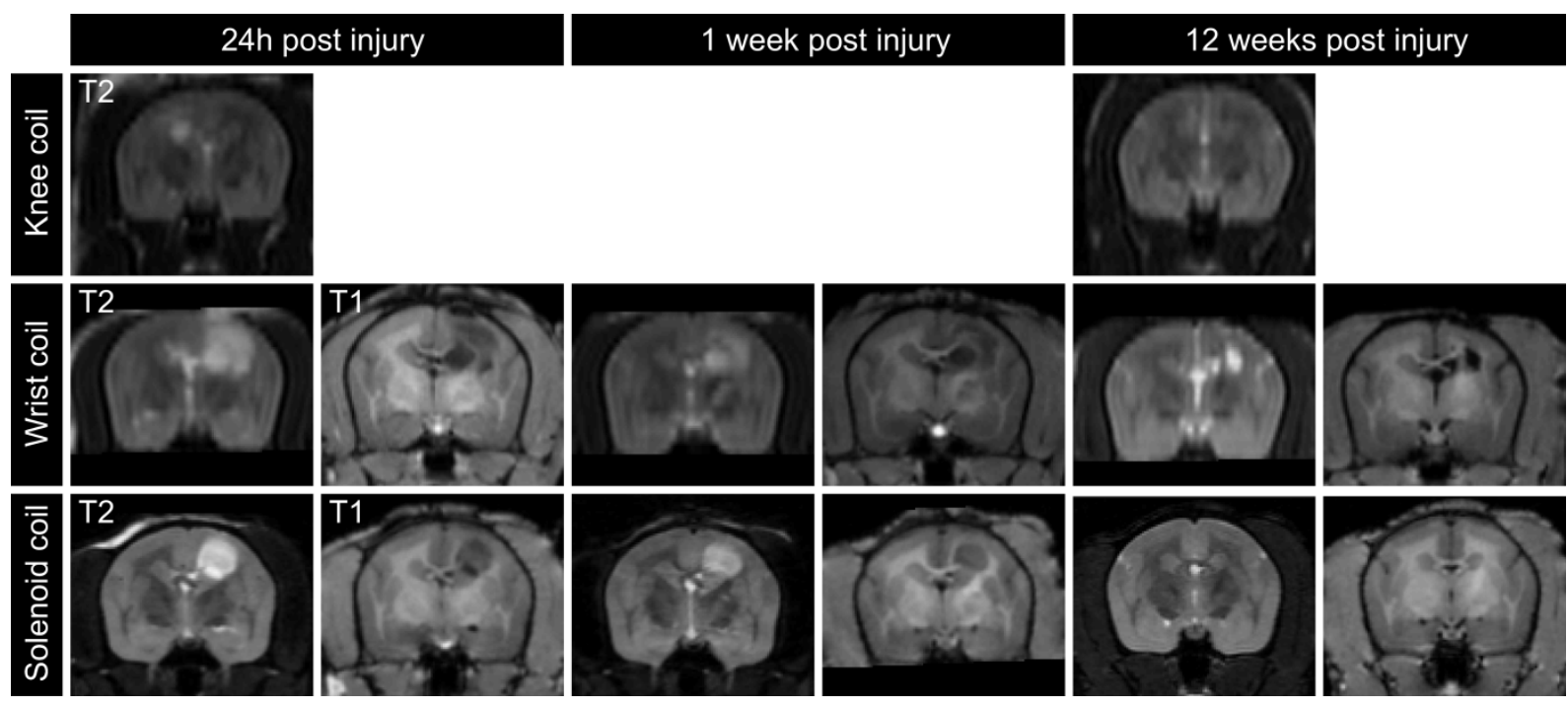

Supplementary figure 1 


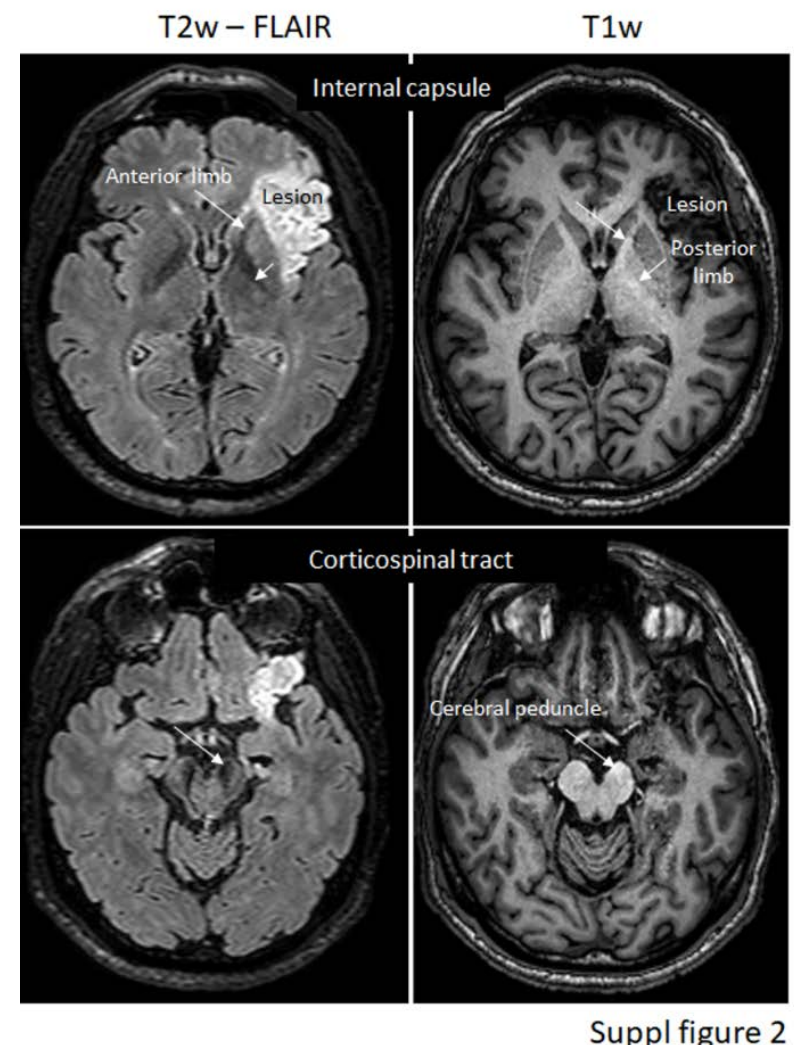

